Portland State University

PDXScholar

\title{
Interstrand Crosslink Resistance in Escherichia coli
}

Jedidiah D. Acott

Portland State University

Follow this and additional works at: https://pdxscholar.library.pdx.edu/honorstheses

\section{Let us know how access to this document benefits you.}

\section{Recommended Citation}

Acott, Jedidiah D., "Interstrand Crosslink Resistance in Escherichia coli" (2018). University Honors Theses. Paper 577.

https://doi.org/10.15760/honors.585

This Thesis is brought to you for free and open access. It has been accepted for inclusion in University Honors Theses by an authorized administrator of PDXScholar. Please contact us if we can make this document more accessible: pdxscholar@pdx.edu. 
Interstrand Crosslink Resistance in Escherichia coli

by

Jedidiah Acott

An undergraduate honors thesis submitted in partial fulfillment of the

Requirements for the degree of

Bachelor of Science

in

University Honors

and

Biology

Thesis Adviser

Justin Courcelle

Portland State University

2018 


\section{Table of Contents}

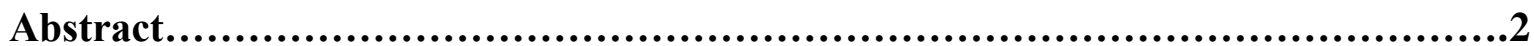

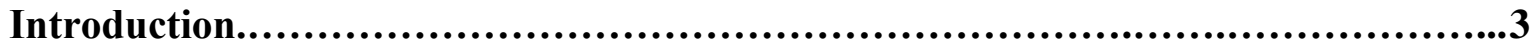

Material and Methods.................................................................... 6

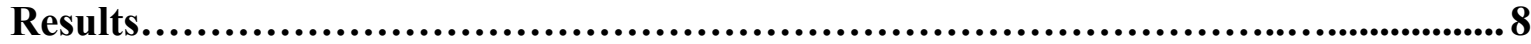

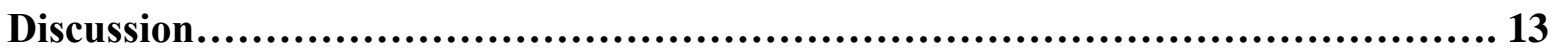

Acknowledgements.................................................................. 17

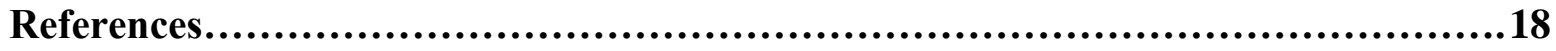




\begin{abstract}
8-methoxypsoralen is a DNA-intercalating agent, which can photoreact with pyrimidine bases on opposing DNA strands, to form an interstrand crosslink. These lesions completely block replication and transcription, and are widely used in chemotherapies; yet how these lesions are processed in the cell remains poorly understood and insight into these processes could lead to better therapies that evade resistance. Previous studies isolated an Escherichia coli mutant demonstrating hyper-resistance to interstrand crosslink-inducing agents. The mutation was mapped to 57.2 minutes on the chromosome, and potentially encoded a $55-\mathrm{kDa}$ protein induced as part of the SOS response. Although these genes remain unidentified, $h s c A$ and $h s c B$ map to this location, have a similar size, and are SOS-inducible. To determine if these or other genes might confer interstrand crosslink resistance in E. coli, we characterized how cells survived 8methoxypsoralen-UVA treatment in the absence of $\mathrm{HscAB}$, and when these gene products were overexpressed. In a second approach, we developed a selection system to isolate hyper-resistant strains through the sequential growth and exposure of wild-type cultures to 8-methoxypsoralenUVA. We found no effect on cell survival in the $h s c A B$ mutant compared to its wild-type parent, suggesting that $\mathrm{HscAB}$ may not contribute to interstrand crosslink resistance as previously hypothesized. However, due to the significant cytotoxicity of plasmids containing $h s c A B$ even in the absence of 8-methoxypsoralen-UVA treatment, we could not determine whether overexpression of these gene products provided cellular protection. Using iteratively 8methoxypsoralen-UVA treated cells, we isolated strains that were $>10^{4}$-fold more resistant to this interstrand crosslink-inducing agent compared to the parent strain. This result suggests that $E$. coli possess mechanisms of interstrand crosslink repair or tolerance and could serve as a model system for understanding the development of drug resistance in human cells.
\end{abstract}




\section{Introduction}

Crosslinking agents are an important class of clinical compounds that have wide use as potent chemotherapeutics and in the treatment of psoriasis and various anemias (Bredberg, Lambert, Lindblad, Swanbeck, \& Wennersten, 1983; Gupta \& Anderson, 1987). Subsequent to cellular internalization, crosslinking agents first intercalate between DNA bases, and then form covalent bonds with cellular DNA through interactions mediated by the reactive functional groups found on these chemicals. Crosslinking agents may form one or multiple covalent interactions, including monoadduct covalent bonds on a single base; intrastrand crosslinks, forming covalent bonds on the same strand; or interstrand crosslinks, forming covalent bonds on opposing DNA strands (Schärer, 2005). The most damaging interaction, interstrand crosslinks, leads to significant cellular toxicity and cell death by preventing the separation of DNA strands during genome replication and transcription. Cisplatin and carboplatin, mitomycin $\mathrm{C}$, and the psoralens are among the most commonly used crosslinking agents in clinics (Guainazzi \& Schärer, 2010). In spite of the successful application of interstrand crosslinking agents in chemotherapy as evidenced by tumor regression, medical professionals have repeatedly documented the emergence of cancers that are resistant to these types of bifunctional drugs (O'Grady et al., 2014). Thus, the development of crosslink resistance in cancer cells represents a major limitation to this therapy and highlights the importance of understanding the cellular mechanisms underlying chemoresistance.

\section{Psoralen as an Interstrand Crosslinking Agents}

Psoralen and its derivatives, have been shown to be effective treatments against the integumentary disorders psoriasis, vitiligo, and cutaneous T-cell lymphoma (Arroyo \& Tift, 2003; Wackernagel, Hofer, Legat, Kerl, \& Wolf, 2006). The three-ring, planar structure of 
psoralen allows the molecule to interact with DNA through intercalation (Cimino, Gamper, Isaacs, \& Hearst, 1985). Thereafter, irradiation with long-wavelength ultraviolet light (UVA) can result in the formation of covalent psoralen-DNA adducts (Cole, 1970; Dall'Acqua, 1977). While psoralens can photo-react with all pyrimidines, these compounds show a preference for thymine particularly in the 5'TpA sequence context (Dall'Acqua, 1977; Kanne, Straub, Rapoport, \& Hearst, 1982). Psoralen-DNA monoadducts are formed through cycloaddition of either the pyrone or furan ring to an adjacent thymine following absorption of one photon of light (Cimino et al., 1985; Kanne et al., 1982). A subset of these monoadducts, furan-side monoadducts, can then be converted to form an interstrand crosslink with a thymine on the opposite DNA strand after absorption of a second photon of UVA light (Sastry, Ross, \& P'arraga, 1997).

\section{DNA Interstrand Crosslink Resistance}

Previous research has implicated multiple mechanisms to explain the observed interstrand crosslink resistance characteristically seen in recurrent post-chemotherapy cancer cells. Many cancers owe their increased survival to the presence of an upregulated ATP-dependent drug pump that may employ reduced cytoplasmic uptake of the crosslinking drugs, increased efflux from the cell, or both (Gottesman, 2002). Downregulation of drug-specific receptors has been observed as well (Cheung-Ong, Giaever, \& Nislow, 2013). Other mechanisms proposed to reduce crosslink formation or increase resistance to crosslinks include increased expression of detoxification genes, and prevention of apoptosis (Huang, Mohanty, \& Basu, 2004; D. Wang \& Lippard, 2005). In addition, enhanced activity of nucleotide excision repair, base excision repair and translesion DNA synthesis enzymes have also been proposed to be involved in resistance to interstrand crosslinks in human cells through either the removal of these replication-blocking 
lesions or their bypass (Ho \& Schärer, 2010; Kaina \& Christmann, 2002; Wilson \& Seidman, 2010).

Previous work by Holland and colleagues suggested that E. coli cells could acquire resistance to interstrand crosslinking agents (Ahmad \& Holland, 1985; Holland, Holland, \& Ahmad, 1991), similar to what has been observed in human cells. This group isolated a mutant strain that displayed increased cell survival to 8-methoxypsoralen-UVA, mitomycin $\mathrm{C}$, and nitrogen mustard treatment compared to a wild-type strain. Resistance to DNA crosslinks was associated with the presence of an overexpressed 55-kDa protein in extracts of this strain, which was inducible by cellular stress. The function of this protein - whether decreased cell permeability, increased crosslink repair, or increased drug pump activity - was not determined, but the gene location was demonstrated to be 57.2 minutes on the E. coli linkage map (Holland et al., 1991).

Given the observed similarities in DNA damage processing between E. coli and eukaryotic cells, we proposed to develop E. coli as a model system for understanding crosslink resistance in cancer cells (Deans \& C West, 2011; Kim \& Wilson, 2012). Here we sought to provide conclusive evidence for the genetic capacity of $E$. coli to acquire resistance to the interstrand crosslinking treatment 8-methoxypsoralen-UVA. Using an analysis of $E$. coli gene expression following UV exposure (J. Courcelle, Khodursky, Peter, Brown, \& Hanawalt, 2001), we found two candidate SOS-inducible genes - hscA and $h s c B$ - located at 57.2 minutes on the E. coli map that could represent the resistance proteins described by Holland and workers (Holland et al., 1991) and examined their role in resistance to 8-methoxypsoralen-UVA. As a second approach to identify novel genes contributing to interstrand crosslink resistance, we also 
developed a selection system to generate $E$. coli that were resistant to 8-methoxypsoralen and UVA exposure.

\section{Materials and Methods}

\section{Bacterial Strains and Plasmids.}

SR108, a thyA36 deoC2 derivative of W3110 (Mellon \& Hanawalt, 1989), was used as the parent for all strains in this study. SR108 recA::Tn10 (HL921) has been previously described (J. Courcelle, Carswell-Crumpton, \& Hanawalt, 1997). SR108 hscAB::cat was constructed in a two-step process. First, the cat cassette was amplified from CL646 (SR108 polB:: $\Omega$ Sm-Spc $\operatorname{din} G:: \operatorname{kan}^{\underline{R}}$ umuDC595::cat, C. T. Courcelle, Belle, \& Courcelle, 2005) using the hscB-catF primer

5'GGATCGCAGCCCTGAGAATGTTATGGATTACTTCACCCTCATGAGACGTTGATCGG CAC and hscA-catR primer 5,

\section{AATAACAATCTTTGGCATATTAAACCTCGTCCACGGAATGCTTTCGAATTTCTGCCAT}

TC. The PCR product was transformed into DY378 (Yu et al., 2000) to generate CL3644, selecting for chloramphenicol resistance. The gene replacement was then moved into SR108 by standard P1 transduction, generating strain CL3679 and CL3680 (SR108 hscAB::cat isolates 1 and 2).

The plasmid pBAD/Myc-HisA was purchased from Invitrogen. To generate pBADHscAB-Myc-HisA, the $h s c A B$ genes were first amplified from SR108 using the pBAD-hscB F primer 5'

CCATACCCGTTTTTTGGGCTAACAGGAGGAATTAACCATGGATTACTTCACCCTCTTT GGC and pBAD-hscA R primer 5' 
CGGCGCTATTCAGATCCTCTTCTGAGATGAGTTTTTGTTCAACCTCGTCCACGGAATG

. The resulting PCR product contained $h s c A B$ genes with flanking sequences homologous to pBAD/Myc-HisA. The pBAD/Myc-HisA vector backbone was amplified from purified plasmid using the pBAD-F primer 5' CATGGTTAATTCCTCCTGTTAGCCCAAAAAACGGGTATGG and pBAD-R primer 5' GAACAAAAACTCATCTCAGAAGAGGATCTGAATAGCGCCG. pBAD-HscAB-Myc-HisA plasmid was generated using Gibson Assembly (NE Biolabs) of equimolar amounts of each PCR product.

SR108 was stably transformed with pBAD/Myc-HisA (pBAD) or pBAD-HscAB-MycHisA (pBAD-HscAB) using standard electroporation procedures.

\section{Psoralen-UV-A survival assays.}

Fresh overnight cultures were grown and diluted 1:100 in Davis media supplemented with $0.4 \%$ glucose, $0.2 \%$ casamino acids, and $10 \mu \mathrm{g} / \mathrm{ml}$ thymine (DGCthy) and grown at $37^{\circ} \mathrm{C}$ to an optical density at $600 \mathrm{~nm}\left(\mathrm{OD}_{600}\right)$ of 0.3 . At this time, $20 \mu \mathrm{g} / \mathrm{ml} 8$-methoxypsoralen was added to the culture and incubated at $37^{\circ} \mathrm{C}$ for 10 minutes. Following incubation, $0.1-\mathrm{ml}$ aliquots of each cultures were removed and serially diluted in 10-fold increments in DGCthy medium. Triplicate $10 \mu \mathrm{l}$ aliquots of each dilution was then spotted on Luria-Bertani agar plates supplemented with $10 \mu \mathrm{g} / \mathrm{ml}$ thymine (LBthy) and $20 \mu \mathrm{g} / \mathrm{ml}$ 8-methoxypsoralen. The cells were then irradiated using two 32-W UV-A bulbs (Sylvania), with a peak emittance of $320 \mathrm{~nm}$ at an incident dose of $6.9 \mathrm{~J} / \mathrm{m}^{2} / \mathrm{s}$ for the indicated doses. Viable colonies were counted following $37^{\circ} \mathrm{C}$ incubation overnight to determine the surviving fraction.

For experiments using $\mathrm{pBAD}$ and $\mathrm{pBAD}-\mathrm{Hsc} \mathrm{AB}$, fresh overnight cultures containing these plasmids were grown in DGCthy medium supplemented with $50 \mu \mathrm{g} / \mathrm{ml}$ ampicillin at $37^{\circ} \mathrm{C}$. The following day, 0.2-ml aliquots of each culture were pelleted to remove ampicillin, diluted 
1:100 in either DGCthy (uninduced) or Davis media supplemented with $0.4 \%$ arabinose, $0.2 \%$ casamino acids, and $10 \mu \mathrm{g} / \mathrm{ml}$ thymine (DACthy, induced) and grown without ampicillin selection at $37^{\circ} \mathrm{C}$ to $\mathrm{OD}_{600}$ of 0.3 . At this time, cultures were treated with $20 \mu \mathrm{g} / \mathrm{ml} 8$ methoxypsoralen for 10 minutes, exposed to varying doses of UVA, and the surviving fraction of cells determined as described above.

\section{Interstrand Crosslink resistance selection.}

A fresh overnight culture of SR108 was grown in LBthy medium at $37^{\circ} \mathrm{C}$. The following day, cells were treated with $20 \mu \mathrm{g} / \mathrm{ml} 8$-methoxypsoralen for 10 minutes, then 0.1 -ml aliquots were plated on LBthy agar plates supplemented with $20 \mu \mathrm{g} / \mathrm{ml}$ 8-methoxypsoralen and subsequently irradiated with UV-A light for the indicated doses. All surviving colonies at a UVA dose producing incremental resistance to 8-methoxypsoralen-UVA treatment were collected the following day and grown in LBthy medium at $37^{\circ} \mathrm{C}$ overnight. Resistant populations were then re-exposed to 8-methoxypsoralen and increasing UVA doses. A portion of culture from each successive selection passage was stored for genome sequencing.

\section{Results}

\section{HscAB does not affect cell survival following 8-methoxypsoralen-UVA-induced DNA damage.}

Previous work by Holland and colleagues identified a 55-kDa that was SOS-inducible, associated with increased resistance to 8-methoxypsoralen-UVA treatment, and mapped to 57.2 minutes on the E. coli genome (Ahmad \& Holland, 1985; Holland et al., 1991). When we examined this region of the genome for genes that encoded proteins of the correct size and whose expression has been reported to be induced by DNA damage (J. Courcelle et al., 2001), we found that $h s c A$ and $h s c B$ fit the criteria. To determine whether HscAB contributes to cell survival 
following 8-methoxypsoralen-UVA treatment, we compared the survival of a $h s c A B$ mutant to that of its wild-type parent. recA mutants, which are known to be sensitive to many forms of DNA damage (Krasin \& Hutchinson, 1977), were also examined as a control. Wild-type cells exhibited decreasing viability when exposed to increasing doses of UVA light in the presence of 8-methoxypsoralen, with approximately $0.1 \%$ of cells surviving UVA doses of greater than 5 $\mathrm{kJ} / \mathrm{m}^{2}$ (Figure 1). recA cells were hypersensitive to UVA exposure in the presence of 8methoxypsoralen, and a UVA dose of $0.5 \mathrm{~kJ} / \mathrm{m}^{2}$ was sufficient to reduce survival by greater than 99.9\%. In contrast, both $h s c A B$ mutant isolates were as sensitive to 8-methoxypsoralen-UVA treatment as the wild-type parent, suggesting that the absence of HscA and HscB does not affect the ability of cells to survive interstrand crosslinks.

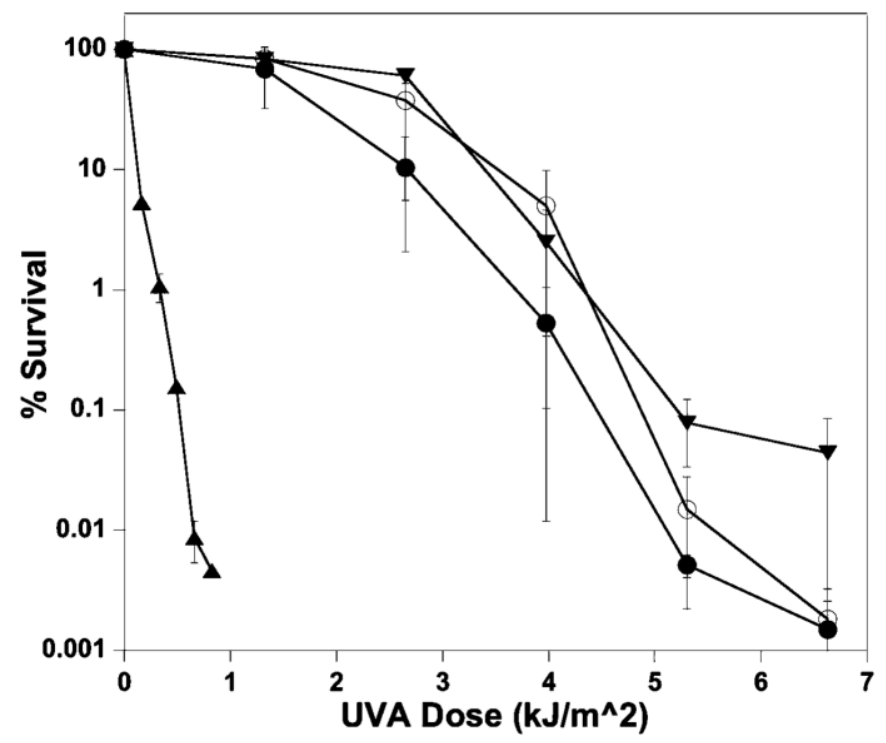

Figure 1. The absence of HscAB does not affect survival following 8-methoxypsoralen-UVAinduced DNA damage. The survival of wild-type (upside-down triangles), recA (triangles), $h s c A B$ isolate 1 (open circles) and $h s c A B$ isolate 2 (filled circles) cells after exposure to $20 \mu \mathrm{g} / \mathrm{ml}$ 8-methoxypsoralen and the indicated doses of UV-A is plotted. Graphs represent an average of two independent experiments. Error bars represent one standard error of the mean. 
While our results suggested $\mathrm{HscA}$ and $\mathrm{HscB}$ do not play a role in cell survival following UVA irradiation in the presence of 8-methoxypsoralen, Holland and colleagues previously hypothesized that interstrand crosslink-resistance might arise from a mutation upregulating expression of a repair protein in the region of $h s c A B$ (Holland et al., 1991). To determine whether overexpression of $h s c A B$ products affects cell survival following 8-methoxypsoralenUVA treatment, we constructed an arabinose-inducible HscAB overexpression plasmid (pBAD$\mathrm{HscAB}$ ), and stably transformed it into wild-type cells. Cells containing pBAD-HscAB were cultured in minimal medium supplemented with arabinose, to induce expression of $\mathrm{HscAB}$, or in minimal medium supplemented with glucose, which silences expression of $\mathrm{HscAB}$ from the plasmid, and then exposed to increasing doses of UVA irradiation in the presence of 8methoxypsoralen. Cells containing the parent vector, $\mathrm{pBAD}$, from which $\mathrm{pBAD}-\mathrm{HscAB}$ was derived, were similarly cultured and exposed to 8-methoxypsoralen with UVA in the same experiment to ensure that any differences we observed were due to HscAB expression.

Cells containing pBAD-HscAB were approximately 100 -fold less viable compared to cells transformed with $\mathrm{pBAD}$, even in the absence of arabinose induction and 8methoxypsoralen-UVA treatment (Figure 2A). This result indicated that HscAB expression from pBAD-HscAB might be leaky and that these proteins could be toxic to cells under normal growth conditions. Unsurprisingly, given this loss in viability in the absence of damage, pBADHscAB containing cells grown in either arabinose- or glucose-supplemented medium were also more sensitive to UVA irradiation in the presence of 8-methoxypsoralen compared to cells containing $\mathrm{pBAD}$ (Figure 2B). This observation further supports the idea that expression of hsc $A B$ products at times outside of the SOS response lowers the viability of cells. From these 
results, we were unable to determine whether upregulation of HscAB expression might enhance resistance to 8-methoxypsoralen-UVA treatment and contribute to interstrand crosslink-repair.

A.

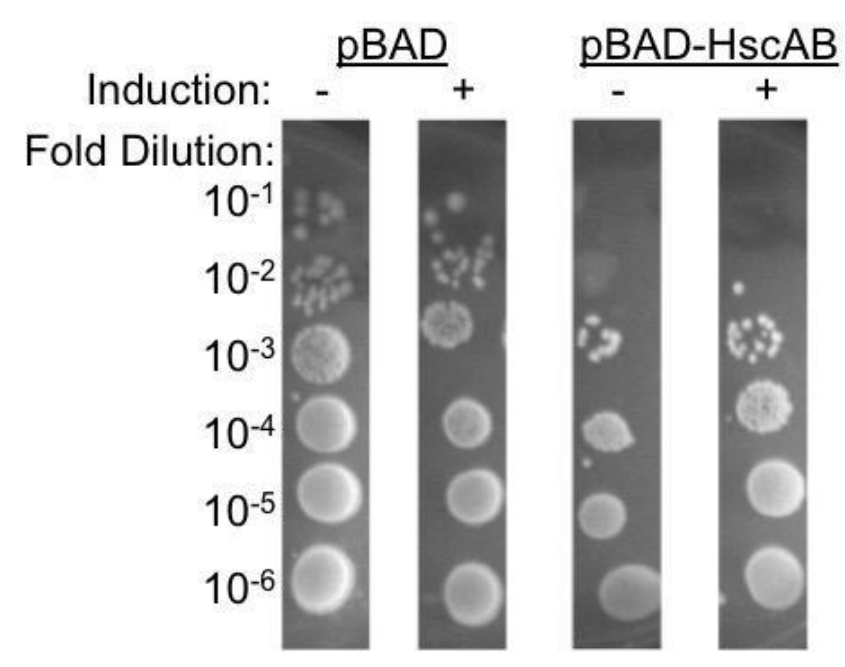

B.

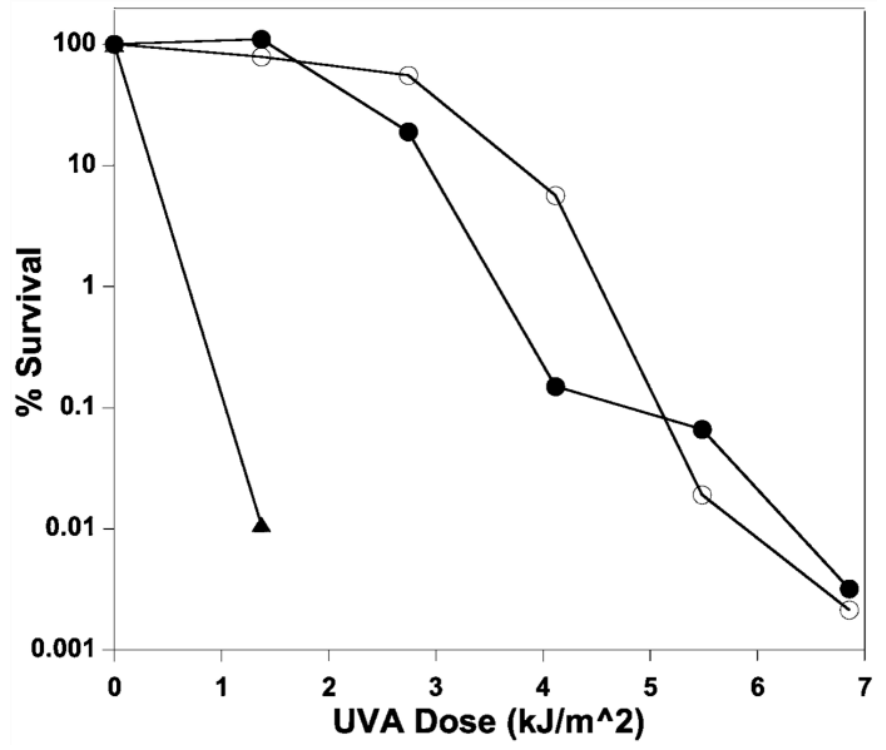

Figure 2. Plasmids containing $h s c A B$ are toxic to cells even in the absence of 8methoxypsoralen-UVA treatment. A) Viability of untreated wild-type cells containing either pBAD-Hsc $\mathrm{AB}$ or $\mathrm{pBAD}$ following growth in glucose- or arabinose-supplemented medium. B) The survival of wild-type cells containing pBAD grown in glucose- (filled circles), pBAD grown in grown in arabinose-supplemented medium (open circles), pBAD-HscAB grown in glucose(filled triangles) and $\mathrm{pBAD}-\mathrm{Hsc} \mathrm{AB}$ grown in arabinose-supplemented medium (open triangles) after exposure to $20 \mu \mathrm{g} / \mathrm{ml} \mathrm{8-methoxypsoralen} \mathrm{and} \mathrm{the} \mathrm{indicated} \mathrm{doses} \mathrm{of} \mathrm{UVA} \mathrm{is} \mathrm{plotted.} \mathrm{The}$ graph represents the results from one independent experiment.

\section{E. coli have the genetic capacity for interstrand crosslink resistance.}

While we were unable to confirm a role for $\mathrm{HscAB}$ in resistance to 8-methoxypsoralen and UVA treatment, it remained possible that $E$. coli encodes alternative genes that confer resistance to interstrand crosslinks. To examine this possibility, we developed a random mutagenesis and functional selection scheme to generate $E$. coli that were resistant to 8- 
methoxypsoralen and UVA exposure. Beginning with wild-type cells, we iteratively exposed cultures to UVA irradiation in the presence of 8-methoxypsoralen, each time selecting for increasingly resistant cells to treat in subsequent generations. For each round of selection, cultures were exposed to incremental doses of UVA irradiation in the presence of 8methoxypsoralen and surviving colonies were then collected in bulk from plates exposed to a UVA dose that produced lethality in most, but not all of the growing cells (Figure 3A). Using nine rounds of this selection protocol, we were able to generate a population of cells that were resistant to and produced a lawn at a UVA dose of $28.32 \mathrm{~kJ} / \mathrm{m}^{2}$ compared to a lawn growth at $3.54 \mathrm{~kJ} / \mathrm{m}^{2} \mathrm{UVA}$ light for the parent strain.

A.

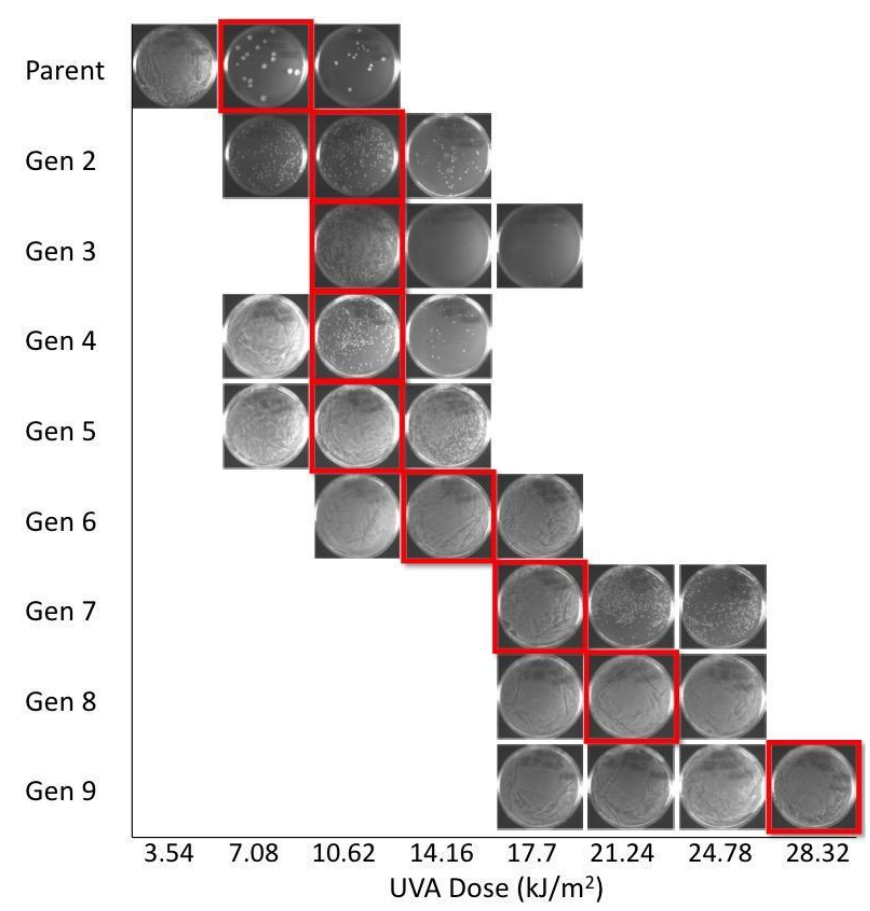

B.

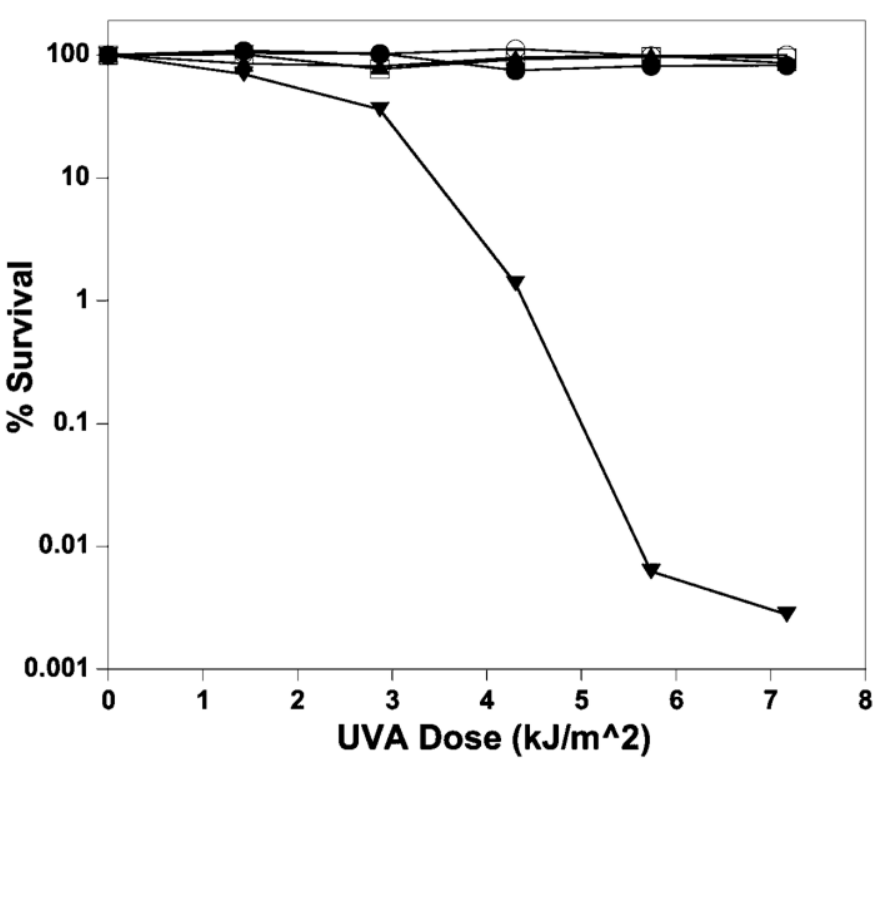

Figure 3. Wild-type cells exposed to successive rounds of 8-methoxypsoralen-UVA treatment develop resistance to this agent. A) Survival of cells exposed to 8-methoxypsoralen-UVA over several rounds of selection. Top row shows viability of parental strain; subsequent rows below show viability over successive generations. Red box denotes the population that was collected, 
propagated and used for selection in the next round. B) The survival of parent (filled triangles), Gen 6 isolate 1 (filled upside-down triangles), Gen 6 isolate 2 (filled circles), Gen 6 isolate 3 (open circles) and Gen 6 isolate 4 (open squares) cells after exposure to $20 \mu \mathrm{g} / \mathrm{ml} 8$ methoxypsoralen and the indicated doses of UVA is plotted. The graph represents the results from one independent experiment.

To directly quantify the resistance of these selected mutants to 8-methoxypsoralen and UVA treatment, we isolated four individuals at random from selection round 6 and examined their ability to survive exposure to this agent. All four individuals from this sixth selection round were hyper-resistant to UVA irradiation in the presence of 8-methoxypsoralen and their viability was unaffected by any of the UVA doses we used in this experiment (Figure 3B). At the highest UVA doses applied ( $6 \mathrm{~kJ} / \mathrm{m}^{2}$ and higher), all four isolates displayed $>10^{4}$-fold increased viability compared to the parent strain. We interpret this result to indicate that E. coli cells have the genetic capacity to develop resistance to crosslink-inducing agents and by extension to interstrand crosslinks.

\section{Discussion}

The purpose of this study was to determine whether E. coli are capable of acquiring resistance to DNA interstrand crosslinks. Using iterative rounds of 8-methoxypsoralen-UVA treatment and selection, I generated cell populations capable of surviving a UVA dose that was $\sim 10$-fold higher than that tolerated by the parent strain. These cells exhibited $>10^{4}$-fold increased resistance by UVA dose compared to their progenitor, suggesting that $E$. coli do indeed contain the genetic capacity to acquire interstrand crosslink resistance.

I was unable to confirm or refute the role of one set of candidate genes implicated in interstrand crosslink resistance by Holland and colleagues (Ahmad \& Holland, 1985; Holland et al., 1991). hscAB mutant cells exhibited no difference in survival to 8-methoxypsoralen-UVA 
treatment compared to wild-type cells, which could be interpreted to mean that these genes have no role in DNA interstrand crosslink resistance or that resistance is dependent on the upregulation of HscAB expression. In the latter case, treating hscAB-deficient cells with UVA irradiation in the presence of 8-methoxypsoralen would be expected to produce a wild-type phenotype such as I observed. I found that cells containing a HscAB-expressing plasmid displayed significant loss in viability even in the absence of treatment, such that the role of Hsc $\mathrm{AB}$ overexpression in interstrand crosslink resistance and repair could not be assessed. It remains possible that $\mathrm{Hsc} \mathrm{AB}$ expression under normal growth conditions confers interstrand crosslink resistance, but assessing these proteins' roles may require more physiologically relevant amounts of protein expression than can be achieved using a plasmid overexpression system. Consistent with this, Holland et al., (1991) proposed that constitutive expression of the 55-kDa product might be dependent on the absence of a repressor protein thus allowing the optimal conditions for DNA interstrand crosslink resistance (Holland et al., 1991). The ironsulfur cluster repressor, IscR, located just downstream of $h s c A B$ may be responsible for providing optimal HscAB concentrations under cellular stress, and could be tested by constructing a iscR-deficient mutant and exposing it to 8-methoxypsoralen-UVA conditions compared to the wild-type strain. Alternatively, HscAB may be involved in multiple cellular pathways beyond interstrand crosslink survival and dysregulation of these genes could lead to cytotoxicity under normal conditions.

Due to the significant cellular toxicity resulting from the inhibition of replication and transcription by interstrand crosslinks in all cells, agents that induce these lesions have been widely adopted as chemotherapeutics and resistance to these covalent linkages have become an important area of study (Deans \& C West, 2011). Our observation that E. coli can acquire 
interstrand crosslink resistance following repeated exposure demonstrates that this bacteria may be a good model system for understanding the development of interstrand crosslink resistance or tolerance over time. If this is the case, the functional homology between metabolic processes in E. coli and mammalian cells would suggest that similar pathways may be exploited by cancer cells to accumulate resistance to crosslinking chemotherapeutic agents over the course of cancer treatment, ultimately leading to cancer recurrence (Guainazzi \& Schärer, 2010).

The exact cellular mechanism responsible for increased cell survival to crosslinking agents remains unknown, but recent studies have implicated enzymes such as Cho endonuclease, along with the UvrAB complex, to be involved in interstrand crosslink repair mechanisms, independent from DNA monoadducts (Perera, Mendenhall, Courcelle, \& Courcelle, 2016). Two alternatively proposed repair mechanisms involve the nucleotide excision repair/lesion bypass (X. Wang et al., 2001); and the nucleotide excision repair/translesion DNA synthesis pathway (Berardini, Foster, \& Loechler, 1999; Kumari et al., 2008). Construction of recA-, uvrA-, polB(DNA Pol II), and $\operatorname{din} B$ (translesion DNA Pol IV)-deficient mutants in the hyper-resistant genetic background may allow for the identification of DNA repair complexes involved in crosslink-lesion processing. If increased repair of 8-methoxypsoralen-bound DNA is responsible for the crosslink-resistant phenotype, then inhibition of the involved complex should cause a reversion in survival towards that seen in repair-deficient mutants.

Another plausible mechanism of the DNA interstrand crosslink resistance observed in this study is transmembrane pump activity, which could remove 8-methoxypsoralen from E. coli cells prior to DNA intercalation and UVA exposure. The multiple drug resistance protein in cancer stem cells has been frequently documented to remove toxic agents from cells prior to DNA alterations, thus contributing to chemotherapy resistance and reduced crosslink formation 
(reviewed in DI \& ZHAO, 2015). In human hepatocytes, induction of crosslinks by 8 methoxypsoralen has been shown to potently induce the oxidative and hydrolytic drug-clearance enzymes cytochrome P450 3A4 and carboxylesterase 2 (Yang \& Yan, 2007). One method to determine whether decreased uptake or increased efflux of 8-methoxypsoralen is responsible for all or some of the hyper-resistance I observed is to monitor the accumulation of DNA interstrand crosslinks over increasing UVA doses. The absence or reduction in accumulation of interstrand crosslinks in the hyper-resistant strain, but not the wild-type cells, would suggest an active transport mechanism as the mode of interstrand crosslink resistance.

This study confirmed that E. coli do possess the capacity for interstrand crosslink resistance within their genomes. Given the functional conservation between cellular processes in E. coli and mammals, identifying which genes are affected in the hyper-resistant strain may provide insights into the cellular pathways involved in DNA interstrand crosslink processing and development of drug resistance. 


\section{Acknowledgements}

This project was funded by the National Institute of Environmental Health Sciences, Grant Number R15ES025953. 


\section{References}

Ahmad, S. I., \& Holland, I. B. (1985). Isolation and analysis of a mutant of Escherichia coli hyper-resistant to near-ultraviolet light plus 8-methoxypsoralen. Mutation Research/Fundamental and Molecular Mechanisms of Mutagenesis, 151(1), 43-47. https://doi.org/10.1016/0027-5107(85)90180-0

Arroyo, M. P., \& Tift, L. (2003). Vitiligo therapy: where are we now? Journal of Drugs in Dermatology: JDD, 2(4), 404-408.

Berardini, M., Foster, P. L., \& Loechler, E. L. (1999). DNA polymerase II (polB) is involved in a new DNA repair pathway for DNA interstrand cross-links in Escherichia coli. Journal of Bacteriology, 181(9), 2878-2882.

Bredberg, A., Lambert, B., Lindblad, A., Swanbeck, G., \& Wennersten, G. (1983). Studies of DNA and Chromosome Damage in Skin Fibroblasts and Blood Lymphocytes from Psoriasis Patients Treated with 8-Methoxypsoralen and UVA Irradiation. Journal of Investigative Dermatology, 81(2), 93-97. https://doi.org/10.1111/1523-1747.ep12542161

Cheung-Ong, K., Giaever, G., \& Nislow, C. (2013). DNA-Damaging Agents in Cancer Chemotherapy: Serendipity and Chemical Biology. Chemistry \& Biology, 20(5), 648659. https://doi.org/10.1016/j.chembiol.2013.04.007

Cimino, G. D., Gamper, H. B., Isaacs, S. T., \& Hearst, J. E. (1985). Psoralens as Photoactive Probes of Nucleic Acid Structure and Function: Organic Chemistry, Photochemistry, and Biochemistry. Annual Review of Biochemistry, 54(1), 1151-1193. https://doi.org/10.1146/annurev.bi.54.070185.005443

Cole, R. S. (1970). Light-induced cross-linking of DNA in the presence of a furocoumarin (psoralen): Studies with phage $\lambda$, Escherichia coli, and mouse leukemia cells. Biochimica 
et Biophysica Acta (BBA) - Nucleic Acids and Protein Synthesis, 217(1), 30-39. https://doi.org/10.1016/0005-2787(70)90119-X

Courcelle, C. T., Belle, J. J., \& Courcelle, J. (2005). Nucleotide Excision Repair or Polymerase V-Mediated Lesion Bypass Can Act To Restore UV-Arrested Replication Forks in Escherichia coli. Journal of Bacteriology, 187(20), 6953-6961. https://doi.org/10.1128/JB.187.20.6953-6961.2005

Courcelle, J., Carswell-Crumpton, C., \& Hanawalt, P. C. (1997). recF and recR are required for the resumption of replication at DNA replication forks in Escherichia coli. Proceedings of the National Academy of Sciences of the United States of America, 94(8), 3714-3719.

Courcelle, J., Khodursky, A., Peter, B., Brown, P. O., \& Hanawalt, P. C. (2001). Comparative gene expression profiles following UV exposure in wild-type and SOS-deficient Escherichia coli. Genetics, 158(1), 41-64.

Dall'Acqua, F. (1977). New Chemical Aspects of the Photoreaction between Psoralen and DNA. In Research in Photobiology (pp. 245-255). Springer, Boston, MA. https://doi.org/10.1007/978-1-4613-4160-4_26

Deans, A., \& C West, S. (2011). DNA interstrand crosslink repair and cancer (Vol. 11). https://doi.org/10.1038/nrc3088

DI, C., \& ZHAO, Y. (2015). Multiple drug resistance due to resistance to stem cells and stem cell treatment progress in cancer (Review). Experimental and Therapeutic Medicine, 9(2), 289-293. https://doi.org/10.3892/etm.2014.2141

Gottesman, M. M. (2002). Mechanisms of Cancer Drug Resistance. Annual Review of Medicine, 53(1), 615-627. https://doi.org/10.1146/annurev.med.53.082901.103929 
Guainazzi, A., \& Schärer, O. D. (2010). Using synthetic DNA interstrand crosslinks to elucidate repair pathways and identify new therapeutic targets for cancer chemotherapy. Cellular and Molecular Life Sciences: CMLS, 67(21), 3683-3697. https://doi.org/10.1007/s00018010-0492-6

Gupta, A. K., \& Anderson, T. F. (1987). Psoralen photochemotherapy. Journal of the American Academy of Dermatology, 17(5), 703-734. https://doi.org/10.1016/S0190$9622(87) 70255-2$

Ho, T. V., \& Schärer, O. D. (2010). Translesion DNA synthesis polymerases in DNA interstrand crosslink repair. Environmental and Molecular Mutagenesis, 51(6), 552-566. https://doi.org/10.1002/em.20573

Holland, J., Holland, I. B., \& Ahmad, S. I. (1991). DNA damage by 8-methoxypsoralen plus near ultraviolet light (PUVA) and its repair in Escherichia coli: Genetic analysis. Mutation Research/DNA Repair, 254(3), 289-298. https://doi.org/10.1016/0921-8777(91)90068-Z

Huang, J., Mohanty, S., \& Basu, A. (2004). Cisplatin resistance is associated with deregulation in protein kinase C- $\delta$. Biochemical and Biophysical Research Communications, 316(4), 1002-1008. https://doi.org/10.1016/j.bbrc.2004.02.149

Kaina, B., \& Christmann, M. (2002). DNA repair in resistance to alkylating anticancer drugs. International Journal of Clinical Pharmacology and Therapeutics, 40(8), 354-367.

Kanne, D., Straub, K., Rapoport, H., \& Hearst, J. E. (1982). The psoralen-DNA photoreaction. Characterization of the monoaddition products from 8-methoxypsoralen and 4,5',8trimethylpsoralen. Biochemistry, 21(5), 861-871. https://doi.org/10.1021/bi00534a008

Kim, Y.-J., \& Wilson, D. M. (2012). Overview of base excision repair biochemistry. Current Molecular Pharmacology, 5(1), 3-13. 
Krasin, F., \& Hutchinson, F. (1977). Repair of DNA double-strand breaks in Escherichia coli, which requires recA function and the presence of a duplicate genome. Journal of Molecular Biology, 116(1), 81-98.

Kumari, A., Minko, I. G., Harbut, M. B., Finkel, S. E., Goodman, M. F., \& Lloyd, R. S. (2008). Replication bypass of interstrand cross-link intermediates by Escherichia coli DNA polymerase IV. The Journal of Biological Chemistry, 283(41), 27433-27437. https://doi.org/10.1074/jbc.M801237200

Mellon, I., \& Hanawalt, P. C. (1989). Induction of the Escherichia coli lactose operon selectively increases repair of its transcribed DNA strand. Nature, 342(6245), 95-98. https://doi.org/10.1038/342095a0

O’Grady, S., Finn, S. P., Cuffe, S., Richard, D. J., O’Byrne, K. J., \& Barr, M. P. (2014). The role of DNA repair pathways in cisplatin resistant lung cancer. Cancer Treatment Reviews, 40(10), 1161-1170. https://doi.org/10.1016/j.ctrv.2014.10.003

Perera, A. V., Mendenhall, J. B., Courcelle, C. T., \& Courcelle, J. (2016). Cho Endonuclease Functions during DNA Interstrand Cross-Link Repair in Escherichia coli. Journal of Bacteriology, 198(22), 3099-3108. https://doi.org/10.1128/JB.00509-16

Sastry, S. S., Ross, B. M., \& P'arraga, A. (1997). Cross-linking of DNA-binding proteins to DNA with psoralen and psoralen furan-side monoadducts. Comparison of action spectra with DNA-DNA cross-linking. The Journal of Biological Chemistry, 272(6), 3715-3723.

Schärer, O. D. (n.d.). DNA Interstrand Crosslinks: Natural and Drug-Induced DNA Adducts that Induce Unique Cellular Responses. ChemBioChem, 6(1), 27-32. https://doi.org/10.1002/cbic.200400287 
Wackernagel, A., Hofer, A., Legat, F., Kerl, H., \& Wolf, P. (2006). Efficacy of 8methoxypsoralen vs. 5-methoxypsoralen plus ultraviolet A therapy in patients with mycosis fungoides. The British Journal of Dermatology, 154(3), 519-523. https://doi.org/10.1111/j.1365-2133.2005.07008.x

Wang, D., \& Lippard, S. J. (2005). Cellular processing of platinum anticancer drugs. Nature Reviews Drug Discovery, 4(4), 307-320. https://doi.org/10.1038/nrd1691

Wang, X., Peterson, C. A., Zheng, H., Nairn, R. S., Legerski, R. J., \& Li, L. (2001). Involvement of Nucleotide Excision Repair in a Recombination-Independent and Error-Prone Pathway of DNA Interstrand Cross-Link Repair. Molecular and Cellular Biology, 21(3), 713-720. https://doi.org/10.1128/MCB.21.3.713-720.2001

Wilson, D. M., \& Seidman, M. M. (2010). A Novel Link to Base Excision Repair. Trends in Biochemical Sciences, 35(5), 247-252. https://doi.org/10.1016/j.tibs.2010.01.003

Yang, J., \& Yan, B. (2007). Photochemotherapeutic agent 8-methoxypsoralen induces cytochrome P450 3A4 and carboxylesterase HCE2: evidence on an involvement of the pregnane X receptor. Toxicological Sciences: An Official Journal of the Society of Toxicology, 95(1), 13-22. https://doi.org/10.1093/toxsci/kfl120

Yu, D., Ellis, H. M., Lee, E.-C., Jenkins, N. A., Copeland, N. G., \& Court, D. L. (2000). An efficient recombination system for chromosome engineering in Escherichia coli. Proceedings of the National Academy of Sciences of the United States of America, 97(11), 5978-5983. 